\title{
H3. Chemotherapy of Brain Tumor by Cerebral Perfusion.
}

\author{
K. TAKEUCHI \\ Dept. of Neurosurg., Toranomon Hospital
}

In 9 cases of malignant brain tumor chemotherapy by selective brain perfusion was performed 10 times during recent one year. Bilateral perfusion was performed in 3 cases. Carcinolytic agents which were heretofore used for perfusion therapy were TESPA $(100-50 \mathrm{mg})$ and Endoxan $(1000 \mathrm{mg})$. Oxygenation of the perfusate was necessary in the normothermic perfusion, whereas it was unnecessary in the hypothermic perfusion which was performed in 3 cases. However, the former is more reasonable from the view point of carcinolytic function of the drugs. Usually perfusion for chemotherapy was continued about 30-40 min.

All but one cases of the Author's series have been well tolerated to the perfusion therapy. One patient of metastatic brain tumor from the pulmonary carcinoma died soon after perfusion by severe cerebral edema which already existed preoperatively. The only and the heaviest side effect was leucopenia and thrombocytopenia due to brain to body leakage of the perfusat. The minimum blood count was usually seen 10-14 days after perfusion.

Postoperatively, buldging of the craniectomized area deminished markedly and neurological signs improved more or less in a few cases. Furthermore, tumor shadow disappeared arteriographically in a case and a marked histological regression was noted in another case.

Though the effect of perfusion therapy heretofore obtained was very poor, chemotherapy of brain tumor by selective cerebral perfusion is promising especially in cases of malignant and surgically unresectable brain tumor.

\section{H4. Treatment of Tumors of Pineal Body.}

\author{
-Irradiation Therapy- \\ K. UekI and Y. SugiYama \\ Dep. of Neurosurg., Univ. of Niigata School of Med.
}

The operative result of tumors of pineal body is not excellent, even hypothermia and hypotensive procedure in use. Therefore radiation thrapy has placd an important part in the treatment. We recognized this fact over again recently and we are taking a course to treat them with irradiation first.

The present series is composed of 31 cases which are 21 cases verified and 10 cases unknown histologically.

Histological verifications are showed in Table 1.

Concerning the symptoms, we would like to point out only some endocrino- 